\title{
GLOBAL CONTINUOUS SOLUTIONS FOR DIAGONAL HYPERBOLIC SYSTEMS WITH LARGE AND MONOTONE DATA
}

\author{
AHMAD EL HAJJ \\ Laboratoire de Mathématiques Appliquées de Compiègne, \\ Université de Technologie de Compiègne, \\ 60205 Compiègne Cedex, France \\ ahmad.el-hajj@utc.fr \\ RÉGIS MONNEAU \\ CERMICS, École Nationale des Ponts et Chaussées, \\ 6 et 8 avenue Blaise Pascal, Cité Descartes, \\ Champs-sur-Marne, 77455 Marne-la-Vallée Cedex 2, France \\ monneau@cermics.enpc.fr
}

Received 27 Mar. 2009

Revised 14 Oct. 2009

Communicated by P. G. LeFloch

\begin{abstract}
In this paper, we study diagonal hyperbolic systems in one space dimension. Based on a new gradient entropy estimate, we prove the global existence of a continuous solution, for large and non-decreasing initial data. We remark that these results cover the case of systems which are hyperbolic but not strictly hyperbolic. Physically, this kind of diagonal hyperbolic system appears naturally in the modelling of the dynamics of dislocation densities.
\end{abstract}

Keywords: Global existence, system of Burgers equations, system of non-linear transport equations, non-linear hyperbolic system, dynamics of dislocation densities.

\section{Introduction and main result}

\subsection{Setting of the problem}

In this paper we are interested in continuous solutions to hyperbolic systems in one dimension. Our work will focus on solutions $u(t, x)=\left(u^{i}(t, x)\right)_{i=1, \ldots, d}$, where $d$ is an integer, of hyperbolic systems which are diagonal, i.e.

$$
\partial_{t} u^{i}+\lambda^{i}(u) \partial_{x} u^{i}=0 \quad \text { on } \quad(0,+\infty) \times \mathbb{R}, \quad \text { for } \quad i=1, \ldots, d,
$$

with the initial data:

$$
u^{i}(0, x)=u_{0}^{i}(x), \quad x \in \mathbb{R}, \quad \text { for } \quad i=1, \ldots, d .
$$


Here $\partial_{t}=\frac{\partial}{\partial t}$ and $\partial_{x}=\frac{\partial}{\partial x}$. Such systems are (sometimes) called $(d \times d)$ hyperbolic systems. Our study of system (1.1) is motivated by the consideration of a model describing the dynamics of dislocation densities (see the Appendix, Section 5), which is given by

$$
\partial_{t} u^{i}+\left(\sum_{j=1, \ldots, d} A_{i j} u^{j}\right) \partial_{x} u^{i}=0 \text { for } i=1, \ldots, d,
$$

where $\left(A_{i j}\right)_{i, j=1, \ldots, d}$ is a non-negative symmetric matrix. This model can be seen as a special case of system (1.1).

For real numbers $\alpha^{i} \leq \beta^{i}$, let us consider the box

$$
U=\Pi_{i=1}^{d}\left[\alpha^{i}, \beta^{i}\right] .
$$

We consider a given function $\lambda=\left(\lambda^{i}\right)_{i=1, \ldots, d}: U \rightarrow \mathbb{R}^{d}$, which satisfies the following regularity assumption:

$$
(H 1) \quad\left\{\begin{array}{l}
\text { the function } \lambda \in C^{\infty}(U), \\
\text { there exists } M_{0}>0 \quad \text { such that for } i=1, \ldots, d, \\
\left|\lambda^{i}(u)\right| \leq M_{0} \quad \text { for all } u \in U, \\
\text { there exists } M_{1}>0 \quad \text { such that for } i=1, \ldots, d, \\
\left|\lambda^{i}(v)-\lambda^{i}(u)\right| \leq M_{1}|v-u| \text { for all } v, u \in U,
\end{array}\right.
$$

where $|w|=\sum_{i=1, \ldots, d}\left|w^{i}\right|$, for $w=\left(w^{1}, \ldots, w^{d}\right)$. Given any Banach space $\left(E,\|\cdot\|_{E}\right)$, throughout this work we define the norm on $E^{d}$ :

$$
\|w\|_{E^{d}}=\sum_{i=1, \ldots, d}\left\|w^{i}\right\|_{E}, \quad \text { for } \quad w=\left(w^{1}, \ldots, w^{d}\right) \in E^{d} .
$$

We assume, for all $u \in \mathbb{R}^{d}$, that the matrix

$$
\left(\lambda_{, j}^{i}(u)\right)_{i, j=1, \ldots, d}, \quad \text { where } \quad \lambda_{, j}^{i}=\frac{\partial \lambda^{i}}{\partial u^{j}},
$$

is non-negative in the positive cone, namely

$$
(H 2) \mid \begin{aligned}
& \text { for all } u \in U, \quad \text { we have } \\
& \sum_{i, j=1, \ldots, d} \xi_{i} \xi_{j} \lambda_{, j}^{i}(u) \geq 0 \quad \text { for every } \xi=\left(\xi_{1}, \ldots, \xi_{d}\right) \in[0,+\infty)^{d} .
\end{aligned}
$$


In (1.2), each component $u_{0}^{i}$ of the initial data $u_{0}=\left(u_{0}^{1}, \ldots, u_{0}^{d}\right)$ is assumed to satisfy the following property:

$$
(H 3) \quad\left\{\begin{array}{l}
\alpha^{i} \leq u_{0}^{i} \leq \beta^{i}, \\
u_{0}^{i} \text { is non-decreasing, } \\
\partial_{x} u_{0}^{i} \in L \log L(\mathbb{R}),
\end{array} \mid \text { for } i=1, \ldots, d,\right.
$$

where $L \log L(\mathbb{R})$ is the following Zygmund space:

$$
L \log L(\mathbb{R})=\left\{f \in L^{1}(\mathbb{R}) \text { such that } \int_{\mathbb{R}}|f| \ln (e+|f|)<+\infty\right\} .
$$

This space is equipped with the following norm:

$$
\|f\|_{L \log L(\mathbb{R})}=\inf \left\{\mu>0: \int_{\mathbb{R}} \frac{|f|}{\mu} \ln \left(e+\frac{|f|}{\mu}\right) \leq 1\right\},
$$

This norm is due to Luxemburg (see Adams [1, (13), Page 234]).

Our purpose is to show the existence of a continuous solution $u=\left(u^{1}, \ldots, u^{d}\right)$ such that, for $i=1, \ldots, d$, the function $u^{i}(t, \cdot)$ satisfies $(H 3)$ for all time.

\subsection{Main result}

It is well-known that for the classical scalar Burgers equation $\partial_{t} u+\partial_{x}\left(\frac{u^{2}}{2}\right)=0$, the solution stays continuous when the initial data is Lipschitz-continuous and non-decreasing. We want somehow to generalize this result to the case of diagonal hyperbolic systems. In particular, we say that a function $u_{0}=\left(u_{0}^{1}, \ldots, u_{0}^{d}\right)$ is non-decreasing if each component $u_{0}^{i}$ is non-decreasing for $i=1, \ldots, d$.

Theorem 1.1. (Global existence of a non-decreasing solution)

Assume (H1), (H2) and (H3). Then, there exists a function $u$ which satisfies for all $T>0$ :

\section{i) Existence of a weak solution:}

The function $u$ is solution of (1.1)-(1.2), where

$u \in\left[L^{\infty}((0,+\infty) \times \mathbb{R})\right]^{d} \cap[C([0,+\infty) ; L \log L(\mathbb{R}))]^{d}$ and $\partial_{x} u \in\left[L^{\infty}((0, T) ; L \log L(\mathbb{R}))\right]^{d}$, such that for a.e. $t \in[0, T)$ the function $u(t, \cdot)$ is non-decreasing in $x$ and satisfies the following $L^{\infty}$ estimate: 


$$
\left\|u^{i}(t, \cdot)\right\|_{L^{\infty}(\mathbb{R})} \leq\left\|u_{0}^{i}\right\|_{L^{\infty}(\mathbb{R})}, \quad \text { for } \quad i=1, \ldots, d,
$$

and the gradient entropy estimate:

$$
\int_{\mathbb{R}} \sum_{i=1, \ldots, d} f\left(\partial_{x} u^{i}(t, x)\right) d x+\int_{0}^{t} \int_{\mathbb{R}_{i, j=1, \ldots, d}} \lambda_{, j}^{i}(u) \partial_{x} u^{i}(s, x) \partial_{x} u^{j}(s, x) d x d s \leq C_{1},
$$

where

$$
0 \leq f(x)= \begin{cases}x \ln (x)+\frac{1}{e} & \text { if } \quad x \geq 1 / e, \\ 0 & \text { if } \quad 0 \leq x \leq 1 / e,\end{cases}
$$

and $C_{1}\left(T, d, M_{1},\left\|u_{0}\right\|_{\left[L^{\infty}(\mathbb{R})\right]^{d}},\left\|\partial_{x} u_{0}\right\|_{[L \log L(\mathbb{R})]^{d}}\right)$.

\section{ii) Continuity of the solution:}

The solution u constructed in (i) belongs to $[C([0,+\infty) \times \mathbb{R})]^{d}$ and there exists a modulus of continuity $\omega(\delta, h)$, such that for all $\delta, h \geq 0$ and all $(t, x) \in(0, T-\delta) \times \mathbb{R}$, we have:

$$
|u(t+\delta, x+h)-u(t, x)| \leq C_{2} \omega(\delta, h) \text { with } \omega(\delta, h)=\frac{1}{\ln \left(\frac{1}{\delta}+1\right)}+\frac{1}{\ln \left(\frac{1}{h}+1\right)},
$$

where $C_{2}\left(T, d, M_{0}, M_{1},\left\|u_{0}\right\|_{\left[L^{\infty}(\mathbb{R})\right]^{d}},\left\|\partial_{x} u_{0}\right\|_{[L \log L(\mathbb{R})]^{d}}\right)$.

The key point to establish Theorem 1.1 is the gradient entropy estimate (1.5). We first consider the parabolic regularization of the system (1.1) and we show that the smooth solution admits the $L^{\infty}$ bound (1.4) and the fundamental gradient entropy inequality (1.5). Then, these a priori estimates will allow us to pass to the limit when the regularization vanishes, which will provide the existence of a solution. Let us mention that a similar gradient entropy inequality was introduced in Cannone et al. [5] to prove the existence of a solution of a two-dimensional system of two coupled transport equations.

Remark 1.2. We remark that assumption $(H 2)$ implies that the second term on the left hand side of (1.5) is non-negative. This will imply the $L \log L$ bound on the gradient of the solutions.

To our knowledge, the result stated in Theorem 1.1 is relatively new. In relation with our result, we cite the work of Poupaud [25], where a result of existence and uniqueness of Lipschitz solutions is proven for a particular quasi-linear hyperbolic system.

Hyperbolic systems (1.1) in the case $d=2$ are called strictly hyperbolic if and only if we have:

$$
\lambda^{1}\left(u^{1}, u^{2}\right)<\lambda^{2}\left(u^{1}, u^{2}\right) .
$$


In this case, a result due to Lax [18] implies the existence of Lipschitz monotone solutions of (1.1)-(1.2). This result was also extended by Serre [26, Vol II] in the case of $(d \times d)$ rich hyperbolic systems (see also Subsection 1.4 for more related references). Their results are limited to the case of strictly hyperbolic systems. On the contrary, in Theorem 1.1, we do not assume that the hyperbolic system is strictly hyperbolic. See the following remark for a quite detailed example.

\section{Remark 1.3. (Crossing eigenvalues)}

Condition (1.8) on the eigenvalues is not required in our framework (Theorem 1.1). Here is a simple example of a $(2 \times 2)$ hyperbolic but not strictly hyperbolic system. We consider solution $u=\left(u^{1}, u^{2}\right)$ of

$$
\left\{\begin{array}{l}
\partial_{t} u^{1}+\cos \left(u^{2}\right) \partial_{x} u^{1}=0, \\
\partial_{t} u^{2}+u^{1} \sin \left(u^{2}\right) \partial_{x} u^{2}=0,
\end{array} \mid \text { on } \quad(0,+\infty) \times \mathbb{R} .\right.
$$

We assume:

i) $u^{1}(-\infty)=1, u^{1}(+\infty)=2$ and $\partial_{x} u^{1} \geq 0$,

ii) $u^{2}(-\infty)=-\frac{\pi}{2}, u^{2}(+\infty)=\frac{\pi}{2}$ and $\partial_{x} u^{2} \geq 0$.

Here the eigenvalues $\lambda^{1}\left(u^{1}, u^{2}\right)=\cos \left(u^{2}\right)$ and $\lambda^{2}\left(u^{1}, u^{2}\right)=u^{1} \sin \left(u^{2}\right)$ cross each other at the initial time (and indeed for any time). Nevertheless, we can compute

$$
\left(\lambda_{, j}^{i}\left(u^{1}, u^{2}\right)\right)_{i, j=1,2}=\left(\begin{array}{cc}
0 & -\sin \left(u^{2}\right) \\
\sin \left(u^{2}\right) & u^{1} \cos \left(u^{2}\right)
\end{array}\right),
$$

which satisfies $(H 2)$ (under assumptions (i) and (ii)). Therefore Theorem 1.1 gives the existence of a solution to (1.9) with in particular (i) and (ii).

\section{Remark 1.4. (A generalization of Theorem 1.1)}

In Theorem 1.1 we have considered a particular system in order to simplify the presentation. Our approach can be easily extended to the following generalized system:

$$
\partial_{t} u^{i}+\lambda^{i}(u, x, t) \partial_{x} u^{i}=h^{i}(u, x, t) \quad \text { on } \quad(0,+\infty) \times \mathbb{R}, \quad \text { for } \quad i=1, \ldots, d,
$$

with the following conditions:

- $\lambda^{i} \in W^{1, \infty}(U \times \mathbb{R} \times[0,+\infty))$ and the matrix $\left(\lambda_{, j}^{i}(u, x, t)\right)_{i, j=1, \ldots, d}$ is positive in the positive cone for all $(u, x, t) \in U \times \mathbb{R} \times[0,+\infty)$ (i.e. a condition analogous to (H2)).

- $h^{i} \in W^{1, \infty}(U \times \mathbb{R} \times[0,+\infty)), \partial_{x} h^{i} \geq 0$ and $h_{, j}^{i} \geq 0$ for all $j \neq i$. 
Let us remark that our system (1.1)-(1.2) does not create shocks because the solution (given in Theorem 1.1) is continuous. In this situation, it seems very natural to expect the uniqueness of the solution. Indeed the notion of entropy solution (in particular designed to deal with the discontinuities of weak solutions) does not seem so helpful in this context. Even for such a simple system, we then ask the following:

Open question: Is there uniqueness of the continuous solution given in Theorem 1.1?

In a companion paper (El Hajj, Monneau [11]), we will provide some partial answers to this question.

\subsection{Application to diagonalizable systems}

Let us first consider a smooth function $u=\left(u^{1}, \ldots, u^{d}\right)$, solution of the following non-conservative hyperbolic system:

$$
\begin{cases}\partial_{t} u(t, x)+F(u) \partial_{x} u(t, x)=0, & u \in U, \quad x \in \mathbb{R}, \quad t \in(0,+\infty), \\ u(x, 0)=u_{0}(x) & x \in \mathbb{R},\end{cases}
$$

where the space of states $U$ is now an open subset of $\mathbb{R}^{d}$, and for each $u, F(u)$ is a $(d \times d)$-matrix and the map $F$ is of class $C^{1}(U)$. The system (1.11) is said to be $(d \times d)$ hyperbolic if $F(u)$ has $d$ real eigenvalues and is diagonalizable for any given $u$ on the domain under consideration. By definition, such a system is said to be diagonalizable if there exists a smooth transformation $w=\left(w^{1}(u), \ldots, w^{d}(u)\right)$ with non-vanishing Jacobian such that (1.11) can be equivalently rewritten (for smooth solutions) as the following system

$$
\partial_{t} w^{i}+\lambda^{i}(w) \partial_{x} w^{i}=0 \quad \text { for } \quad i=1, \ldots, d,
$$

where $\lambda^{i}$ are smooth functions of $w$. Such functions $w^{i}$ are called strict $i$-Riemann invariant.

Our approach can give continuous solutions to the diagonalized system, which provides a continuous solution to the original system (1.11).

\subsection{A brief review of some related literature}

For a scalar conservation law, which corresponds to system (1.11) in the case $d=1$ where $F(u)=h^{\prime}(u)$ is the derivative of some flux function $h$, the global existence and uniqueness of $B V$ solutions has been established by Oleinik [24] in one space dimension. The famous paper of Kruzhkov [17] covers the more general class of $L^{\infty}$ solution in several space dimensions. For an alternative approach based on the notion of entropy process solutions see for instance Eymard et al. [12]. For a different approach based on a kinetic formulation, see also Lions et al. [23]. 
We now recall some well-known results for a class of $(2 \times 2)$ strictly hyperbolic systems in one space dimension. This means that $F(u)$ has two real, distinct eigenvalues satisfying (1.8). As mentioned above, Lax [18] proved the existence and uniqueness of non-decreasing and smooth solutions for diagonalized $(2 \times 2)$ strictly hyperbolic systems. In the case of some $(2 \times 2)$ strictly hyperbolic systems, DiPerna [6,7] showed the global existence of a $L^{\infty}$ solution. The proof of DiPerna relies on a compensated compactness argument based on the representation of the weak limit in terms of Young measures which must reduce to a Dirac mass due to the presence of a large family of entropies. This result is based on an the idea of Tartar [28].

For general $(d \times d)$ strictly hyperbolic systems; i.e. where $F(u)$ has $d$ real, distinct eigenvalues

$$
\lambda^{1}(u)<\cdots<\lambda^{d}(u)
$$

Bianchini and Bressan proved in a very complete paper [3] a striking global existence and uniqueness result of solutions to system (1.11) assuming that the initial data has small total variation. This approach is mainly based on a careful analysis of the vanishing viscosity approximation. An existence result has first been proved by Glimm [14] in the special case of conservative equations, i.e. $F(u)=D h(u)$ is the Jacobian of some flux function $h$. Let us mention that an existence result has been also obtained by LeFloch, Liu [20] and LeFloch [19,21], in the non-conservative case.

We can also mention that our system (1.1) is related to other similar models with dimension $N \geq 1$, such as scalar transport equations based on vector fields with low regularity. Such equations were for instance studied by Diperna and Lions in [8]. They have proved the existence (and uniqueness) of a solution (in the renormalized sense), for given vector fields in $L^{1}\left((0,+\infty) ; W_{l o c}^{1,1}\left(\mathbb{R}^{N}\right)\right)$ whose divergence is in $L^{1}\left((0,+\infty) ; L^{\infty}\left(\mathbb{R}^{N}\right)\right)$. This study was generalized by Ambrosio [2], who considered vector fields in $L^{1}\left((0,+\infty) ; B V_{l o c}\left(\mathbb{R}^{N}\right)\right)$ with bounded divergence. In the present paper, we work in dimension $N=1$ and prove the existence (and some uniqueness results) of solutions of the system (1.1)-(1.2) with a velocity vector field $\lambda^{i}(u), i=1, \ldots, d$. Here, in Theorem 1.1, the divergence of our vector field is only in $L^{\infty}((0,+\infty), L \log L(\mathbb{R}))$. In this case we proved the existence result thanks to the gradient entropy estimate (1.5), which gives a better estimate on the solution.

Let us also mention that for hyperbolic and symmetric systems in dimension $N \geq 1$, Garding has proved in [13] a local existence and uniqueness result in $C\left([0, T) ; H^{s}\left(\mathbb{R}^{N}\right)\right) \cap C^{1}\left([0, T) ; H^{s-1}\left(\mathbb{R}^{N}\right)\right.$ ), with $s>\frac{N}{2}+1$ (see also Serre [26, Vol I, Th 3.6.1]), this result being only local in time even in dimension $N=1$. 


\subsection{Organization of the paper}

This paper is organized as follows: in Section 2, we approximate the system (1.1) by adding the viscosity term $\left(\varepsilon \partial_{x x} u^{i}\right)$. Then we show a global in time existence for this approximated system. Moreover, we show that these solutions are regular and nondecreasing in $x$ for all $t>0$. In Section 3, we prove the gradient entropy inequality and some other $\varepsilon$-uniform a priori estimates. In Section 4 , we prove the main result (Theorem 1.1) passing to the limit as $\varepsilon$ goes to 0 . Finally, in the appendix (Section 5 ), we derive a model for the dynamics of dislocation densities.

\section{Local existence of an approximated system}

The system (1.1) can be written as:

$$
\partial_{t} u+\lambda(u) \diamond \partial_{x} u=0
$$

where $u:=\left(u^{i}\right)_{1, \ldots, d}, \lambda(u)=\left(\lambda^{i}(u)\right)_{1, \ldots, d}$ and $u \diamond v$ is the "component by component product" of the two vectors $u=\left(u^{1}, \ldots, u^{d}\right), v=\left(v^{1}, \ldots, v^{d}\right) \in \mathbb{R}^{d}$. This is the vector in $\mathbb{R}^{d}$ whose coordinates are given by $(u \diamond v)^{i}:=u^{i} v^{i}$. We now consider the following parabolic regularization of system (2.1), for all $0<\varepsilon \leq 1$ :

$$
\left\{\begin{array}{l}
\partial_{t} u^{\varepsilon}+\lambda\left(u^{\varepsilon}\right) \diamond \partial_{x} u^{\varepsilon}=\varepsilon \partial_{x x} u^{\varepsilon} \\
u^{\varepsilon}(x, 0)=u_{0}^{\varepsilon}(x), \quad \text { with } \quad u_{0}^{\varepsilon}(x):=u_{0} * \eta_{\varepsilon}(x),
\end{array}\right.
$$

where $\partial_{x x}=\frac{\partial^{2}}{\partial x^{2}}$ and $\eta_{\varepsilon}$ is a mollifier verifying $\eta_{\varepsilon}(\cdot)=\frac{1}{\varepsilon} \eta(\dot{\bar{\varepsilon}})$, such that $\eta \in C_{c}^{\infty}(\mathbb{R})$ is a non-negative function satisfying $\int_{\mathbb{R}} \eta=1$.

Remark 2.1. By classical properties of the mollifier $\left(\eta_{\varepsilon}\right)_{\varepsilon}$ and the fact that $u_{0}^{\varepsilon} \in$ $\left[L^{\infty}(\mathbb{R})\right]^{d}$, then $u_{0} \in\left[C^{\infty}(\mathbb{R})\right]^{d} \cap\left[W^{2, \infty}(\mathbb{R})\right]^{d}$. Moreover using the non-negativity of $\eta_{\varepsilon}$, the second equation of $(2.2)$ gives

$$
\left\|u_{0}^{\varepsilon, i}\right\|_{L^{\infty}(\mathbb{R})} \leq\left\|u_{0}^{i}\right\|_{L^{\infty}(\mathbb{R})}, \quad \text { for } \quad i=1, \ldots, d,
$$

and (H3) also implies that $u_{0}^{\varepsilon}$ is non-decreasing.

The following theorem is a global existence result for the regularized system (2.2).

Theorem 2.2. (Global existence of non-decreasing smooth solutions)

Assume $(H 1)$ and that the initial data $u_{0}^{\varepsilon}$ is non-decreasing and satisfies $u_{0}^{\varepsilon} \in\left[C^{\infty}(\mathbb{R})\right]^{d} \cap\left[W^{2, \infty}(\mathbb{R})\right]^{d}$. Then the system (2.2) admits a solution $u^{\varepsilon} \in$ $\left[C^{\infty}([0,+\infty) \times \mathbb{R})\right]^{d} \cap\left[W^{2, \infty}((0,+\infty) \times \mathbb{R})\right]^{d}$ such that the function $u^{\varepsilon}(t, \cdot)$ is nondecreasing for all $t>0$. Moreover, for all $t>0$, we have the a priori bounds:

$$
\left\|u^{\varepsilon, i}(t, \cdot)\right\|_{L^{\infty}(\mathbb{R})} \leq\left\|u_{0}^{\varepsilon, i}\right\|_{L^{\infty}(\mathbb{R})}, \quad \text { for } \quad i=1, \ldots, d,
$$




$$
\left\|\partial_{x} u^{\varepsilon, i}\right\|_{L^{\infty}\left([0,+\infty) ; L^{1}(\mathbb{R})\right)} \leq 2\left\|u_{0}^{\varepsilon, i}\right\|_{L^{\infty}(\mathbb{R})}, \quad \text { for } \quad i=1, \ldots, d .
$$

The lines of the proof of this theorem are very standard (see for instance Cannone et al. [5] for a similar problem). For this reason, we skip the details of the proof. First of all, we remark that the estimate (2.3) is a direct application of the Maximum Principle Theorem for parabolic equations (see Lieberman [22, Th.2.10]). The regularity of the solution follows from a bootstrap argument. The monotonicity of the solution is a consequence of the maximum principle for scalar parabolic equations applied to $w^{\varepsilon}=\partial_{x} u^{\varepsilon}$ satisfying

$$
\partial_{t} w^{\varepsilon}+\lambda\left(u^{\varepsilon}\right) \diamond \partial_{x} w^{\varepsilon}+\partial_{x}\left(\lambda\left(u^{\varepsilon}\right)\right) \diamond w^{\varepsilon}=\varepsilon \partial_{x x} w^{\varepsilon} .
$$

Since $\partial_{x} u^{\varepsilon} \geq 0$ this easily implies the second estimate (2.4).

\section{3. $\varepsilon$-uniform a priori estimates}

In this section, we show some $\varepsilon$-uniform estimates on the solutions of system (2.2).

Before demonstrating the proof of the gradient entropy inequality defined in (1.5), we announce the main idea to establish this estimate. Now, let us set for $w \geq 0$ the entropy function

$$
\bar{f}(w)=w \ln w .
$$

For any non-negative test function $\varphi \in C_{c}^{1}([0,+\infty) \times \mathbb{R})$, let us define the following "gradient entropy" with $w^{i}:=\partial_{x} u^{i}$ :

$$
\bar{S}(t)=\int_{\mathbb{R}} \varphi(t, \cdot)\left(\sum_{i=1, \ldots, d} \bar{f}\left(w^{i}(t, \cdot)\right)\right) d x
$$

It is very natural to introduce the quantity $\bar{S}(t)$ which in the case $\varphi \equiv 1$ appears to be nothing else than the total entropy of the system of $d$ types of particles of non-negative densities $w^{i} \geq 0$. Then after two integration by parts, it is formally possible to deduce from (1.1) the equality in the following gradient entropy inequality for all $t \geq 0$

$$
\frac{d \bar{S}(t)}{d t}+\int_{\mathbb{R}} \varphi\left(\sum_{i, j=1, \ldots, d} \lambda_{, j}^{i} w^{i} w^{j}\right) d x \leq R(t), \quad \text { for } \quad t \geq 0,
$$

with the rest

$$
R(t)=\int_{\mathbb{R}}\left\{\left(\partial_{t} \varphi\right)\left(\sum_{i=1, \ldots, d} \bar{f}\left(w^{i}\right)\right)+\left(\partial_{x} \varphi\right)\left(\sum_{i=1, \ldots, d} \lambda^{i} \bar{f}\left(w^{i}\right)\right)\right\} d x
$$


where we do not show the dependence on $t$ in the integrals. We remark in particular that the rest is formally equal to zero if $\varphi \equiv 1$.

To guarantee the existence of continuous solutions when $\varepsilon=0$, we will assume later $(H 2)$ which guarantees the non-negativity on the second term of the left hand side of inequality (3.1).

Returning to a rigorous statement, we will prove the following result.

Proposition 3.1. (Gradient entropy inequality)

Assume (H1) and consider a function $u_{0} \in\left[L^{\infty}(\mathbb{R})\right]^{d}$ satisfying (H3). For any $0<\varepsilon \leq 1$, we consider the solution $u^{\varepsilon}$ of the system (2.2) given in Theorem 2.2 with initial data $u_{0}^{\varepsilon}=u_{0} * \eta_{\varepsilon}$. Then for any $T>0$, there exists a constant $C\left(T, d, M_{1},\left\|u_{0}\right\|_{\left[L^{\infty}(\mathbb{R})\right]^{d}},\left\|\partial_{x} u_{0}\right\|_{[L \log L(\mathbb{R})]^{d}}\right)$ such that

$S(t)+\int_{0}^{t} \int_{\mathbb{R}_{i, j=1, \ldots, d}} \lambda_{, j}^{i}\left(u^{\varepsilon}\right) w^{\varepsilon, i} w^{\varepsilon, j} \leq C, \quad$ with $\quad S(t)=\int_{\mathbb{R}_{i=1, \ldots, d}} \sum_{i} f\left(w^{\varepsilon, i}(t, \cdot)\right) d x$.

where $f$ is defined in (1.6) and $w^{\varepsilon}=\left(w^{\varepsilon, i}\right)_{i=1, \ldots, d}=\partial_{x} u^{\varepsilon}$.

For the proof of Proposition 3.1, we need the following technical lemma:

Lemma 3.2. (L $\log L$ estimate)

Let $\left(\eta_{\varepsilon}\right)_{\varepsilon \in(0,1]}$ be a non-negative mollifier satisfying $\int_{\mathbb{R}} \eta_{\varepsilon}=1$, let $f$ be the function defined in (1.6) and $h \in L^{1}(\mathbb{R})$ be a non-negative function. Then

i) $\int_{\mathbb{R}} f(h)<+\infty$ if and only if $h \in L \log L(\mathbb{R})$. Moreover we have the following estimates:

$$
\begin{gathered}
\int_{\mathbb{R}} f(h) \leq 1+\|h\|_{L \log L(\mathbb{R})}+\|h\|_{L^{1}(\mathbb{R})} \ln \left(1+\|h\|_{L \log L(\mathbb{R})}\right), \\
\|h\|_{L \log L(\mathbb{R})} \leq 1+\int_{\mathbb{R}} f(h)+\ln \left(1+e^{2}\right)\|h\|_{L^{1}(\mathbb{R})} .
\end{gathered}
$$

ii) If $h \in L \log L(\mathbb{R})$, then for every $\varepsilon \in(0,1]$ the function $h_{\varepsilon}=h * \eta_{\varepsilon} \in L \log L(\mathbb{R})$ and satisfies

$$
\left\|h_{\varepsilon}\right\|_{L \log L(\mathbb{R})} \leq C\|h\|_{L \log L(\mathbb{R})} \quad \text { and } \quad\left\|h-h_{\varepsilon}\right\|_{L \log L(\mathbb{R})} \rightarrow 0 \quad \text { as } \quad \varepsilon \rightarrow 0,
$$

where $C$ is a universal constant.

\section{Proof of Lemma 3.2:}

The proof of (i) is trivial. To prove estimate (3.3), we first remark that for all $h \geq 0$ and $\mu \in(0,1]$, we have 


$$
\left(h \ln (h)+\frac{1}{e}\right) \mathbb{1}_{\left\{h \geq \frac{1}{e}\right\}} \leq h \ln (h+e) \leq h \ln (e+\mu h)+|\ln (\mu)| h .
$$

We apply this inequality with $\mu=\frac{1}{\max \left(1,\|h\|_{L \log L(\mathbb{R})}\right)}$ and integrate, we get

$$
\begin{aligned}
\int_{\mathbb{R}} f(h) & \leq \frac{1}{\mu} \int_{\mathbb{R}} \mu h \ln (e+\mu h)+|\ln (\mu)|\|h\|_{L^{1}(\mathbb{R})} \\
& \leq \frac{1}{\mu}+|\ln (\mu)|\|h\|_{L^{1}(\mathbb{R})},
\end{aligned}
$$

where we have used the definition of $\|h\|_{L \log L(\mathbb{R})}$. This gives (3.3) using the fact that $\mu \geq \frac{1}{1+\|h\|_{L \log L(\mathbb{R})}}$.

To prove (3.4), we remark that for $h \geq \frac{1}{e}$, we have $e \leq e^{2} h$ and

$$
h \ln (e+h) \leq h \ln (h)+h \ln \left(1+e^{2}\right) \leq f(h)+h \ln \left(1+e^{2}\right) .
$$

However, for $0 \leq h \leq \frac{1}{e}$, we have in particular that

$$
h \ln (e+h) \leq h \ln \left(1+e^{2}\right),
$$

and therefore

$$
\int_{\mathbb{R}} h \ln (e+h) \leq \int_{\mathbb{R}} f(h)+\ln \left(1+e^{2}\right)\|h\|_{L^{1}(\mathbb{R})} .
$$

From the definition of $\|h\|_{L \log L(\mathbb{R})}$, we deduce in particular (3.4). For the proof of (ii) see Adams [1, Th 8.20].

\section{Proof of Proposition 3.1:}

First we want to check that $S(t)$ is well defined. To this end, we remark that if $w \geq 0$, then

$$
0 \leq f(w) \leq \frac{1}{e} \mathbb{1}_{\left\{w \geq \frac{1}{e}\right\}}+w \ln (1+w)
$$

Which gives that

$$
\int_{\mathbb{R}} f(w) \leq\|w\|_{L^{1}(\mathbb{R})} \ln \left(1+\|w\|_{L^{\infty}(\mathbb{R})}\right)+\int_{\mathbb{R}} \frac{1}{e} \mathbb{1}_{\left\{w \geq \frac{1}{e}\right\}} \leq\|w\|_{L^{1}(\mathbb{R})}\left(1+\ln \left(1+\|w\|_{L^{\infty}(\mathbb{R})}\right)\right) .
$$

Now by Theorem 2.2, we have $\partial_{x} u^{\varepsilon}=w^{\varepsilon} \in\left[L^{\infty}\left((0,+\infty) ; L^{1}(\mathbb{R})\right)\right]^{d} \cap$ $\left[W^{2, \infty}((0,+\infty) \times \mathbb{R})\right]^{d}$. This implies that $S \in L^{\infty}(0,+\infty)$. We compute 


$$
\begin{aligned}
\frac{d}{d t} S(t) & =\int_{\mathbb{R}} \sum_{i=1, \ldots, d} f^{\prime}\left(w^{\varepsilon, i}\right)\left(\partial_{t} w^{\varepsilon, i}\right), \\
& =\int_{\mathbb{R}_{i=1, \ldots, d}} f^{\prime}\left(w^{\varepsilon, i}\right) \partial_{x}\left(-\lambda^{i}\left(u^{\varepsilon}\right) w^{\varepsilon, i}+\varepsilon \partial_{x} w^{\varepsilon, i}\right), \\
& =\overbrace{\int_{\mathbb{R}} \sum_{i=1, \ldots, d} \lambda^{i}\left(u^{\varepsilon}\right) w^{\varepsilon, i} f^{\prime \prime}\left(w^{\varepsilon, i}\right) \partial_{x} w^{\varepsilon, i}}^{J_{1}} \overbrace{-\varepsilon \int_{\mathbb{R}} \sum_{i=1, \ldots, d}\left(\partial_{x} w^{\varepsilon, i}\right)^{2} f^{\prime \prime}\left(w^{\varepsilon, i}\right)}^{J_{2}} .
\end{aligned}
$$

We note that these computations (and the integration by parts) are justified because on the one hand $w^{\varepsilon, i}$, its derivatives and $\lambda^{i}$ are bounded, and on the other hand $w^{\varepsilon, i}$ is in $L^{\infty}\left((0,+\infty) ; L^{1}(\mathbb{R})\right)$. We know that $J_{2} \leq 0$ because $f$ is convex. To control $J_{1}$, we rewrite it under the following form

$$
J_{1}=\int_{\mathbb{R}_{i=1, \ldots, d}} \lambda^{i}\left(u^{\varepsilon}\right) g^{\prime}\left(w^{\varepsilon, i}\right) \partial_{x} w^{\varepsilon, i}
$$

where

$$
g(x)= \begin{cases}x-\frac{1}{e} & \text { if } \quad x \geq 1 / e \\ 0 & \text { if } \quad 0 \leq x \leq 1 / e .\end{cases}
$$

Then, we deduce that

$$
\begin{aligned}
J_{1} & =\int_{\mathbb{R}_{i=1, \ldots, d}} \lambda^{i}\left(u^{\varepsilon}\right) \partial_{x}\left(g\left(w^{\varepsilon, i}\right)\right) \\
& =-\int_{\mathbb{R}_{i, j=1, \ldots, d} \sum_{, j} \lambda^{i}\left(u^{\varepsilon}\right) w^{\varepsilon, j} g\left(w^{\varepsilon, i}\right)} \overbrace{}^{J_{11}} \overbrace{-\int_{\mathbb{R}_{i, j=1, \ldots, d}} \lambda_{, j}^{i}\left(u^{\varepsilon}\right) w^{\varepsilon, j} w^{\varepsilon, i}}^{J_{12}} \overbrace{-\int_{\mathbb{R}_{i, j=1, \ldots, d}} \lambda_{, j}^{i}\left(u^{\varepsilon}\right) w^{\varepsilon, j}\left(g\left(w^{\varepsilon, i}\right)-w^{\varepsilon, i}\right)}^{J_{12}} .
\end{aligned}
$$

We use the fact that $|g(x)-x| \leq \frac{1}{e}$ for all $x \geq 0$ and (H1), to deduce that

$$
\begin{aligned}
\left|J_{12}\right| & \leq \frac{1}{e} d M_{1}\left\|w^{\varepsilon}\right\|_{\left[L^{\infty}\left((0,+\infty), L^{1}(\mathbb{R})\right)\right]^{d}} \\
& \leq \frac{2}{e} d M_{1}\left\|u_{0}\right\|_{\left[L^{\infty}(\mathbb{R})\right]^{d}}:=C_{0}\left(\left\|u_{0}\right\|_{\left[L^{\infty}(\mathbb{R})\right]^{d}}, d, M_{1}\right)
\end{aligned}
$$

where we have used 


$$
\left\|w^{\varepsilon, i}\right\|_{L^{\infty}\left((0,+\infty), L^{1}(\mathbb{R})\right)} \leq 2\left\|u_{0}^{i}\right\|_{L^{\infty}(\mathbb{R})}, \quad \text { for } \quad i=1, \ldots, d,
$$

which follows from Remark 2.1 and Theorem 2.2. Finally, we deduce that

$$
\begin{aligned}
\frac{d}{d t} S(t) & \leq J_{11}+J_{12}+J_{2} \\
& \leq J_{11}+C_{0}
\end{aligned}
$$

Integrating in time on $(0, t)$, for $0<t<T$, we get that there exists a positive constant $C\left(T, d, M_{1},\left\|u_{0}\right\|_{\left[L^{\infty}(\mathbb{R})\right]^{d}},\left\|\partial_{x} u_{0}\right\|_{[L \log L(\mathbb{R})]^{d}}\right)$ which is independent of $\varepsilon$ by (3.3) and Lemma 3.2 (ii) such that

$$
S(t)+\int_{0}^{t} \int_{\mathbb{R}_{i, j=1, \ldots, d}} \lambda_{, j}^{i}\left(u^{\varepsilon}\right) w^{\varepsilon, j} w^{\varepsilon, i} \leq C_{0} T+S(0) \leq C .
$$

Lemma 3.3. ( $W^{-1,1}$ estimate on the time derivative of the solutions) Assume (H1) and that the function $u_{0} \in\left[L^{\infty}(\mathbb{R})\right]^{d}$ satisfies (H3). Then for any $0<\varepsilon \leq 1$, the solution $u^{\varepsilon}$ of the system (2.2) given in Theorem 2.2 with initial data $u_{0}^{\varepsilon}=u_{0} * \eta_{\varepsilon}$, satisfies the following $\varepsilon$-uniform estimate for all $T>0$ :

$$
\left\|\partial_{t} u^{\varepsilon}\right\|_{\left[L^{2}\left((0, T) ; W^{-1,1}(\mathbb{R})\right)\right]^{d}} \leq C\left\|u_{0}\right\|_{\left[L^{\infty}(\mathbb{R})\right]^{d}} .
$$

where $C=C\left(T, M_{0}\right)>0$ and $W^{-1,1}(\mathbb{R})$ is the dual of the space $W^{1, \infty}(\mathbb{R})$.

\section{Proof of Lemma 3.3:}

The idea to bound $\partial_{t} u^{\varepsilon}$ is simply to use the available bounds on the right hand side of the equation (2.2). We will give a proof by duality. We multiply the equation (2.2) by $\phi \in\left[L^{2}\left((0, T), W^{1, \infty}(\mathbb{R})\right)\right]^{d}$ and integrate on $(0, T) \times \mathbb{R}$, which gives

$$
\int_{(0, T) \times \mathbb{R}} \phi \cdot \partial_{t} u^{\varepsilon}=\overbrace{\varepsilon \int_{(0, T) \times \mathbb{R}} \phi \cdot \partial_{x x}^{2} u^{\varepsilon}}^{I_{1}} \overbrace{-\int_{(0, T) \times \mathbb{R}} \phi \cdot\left(\lambda\left(u^{\varepsilon}\right) \diamond \partial_{x} u^{\varepsilon}\right)}^{I_{2}} .
$$

We integrate by parts term $I_{1}$, and obtain:

$$
\begin{aligned}
\left|I_{1}\right| \leq\left|\int_{(0, T) \times \mathbb{R}} \partial_{x} \phi \cdot \partial_{x} u^{\varepsilon}\right| & \leq\left\|\partial_{x} \phi\right\|_{\left[L^{2}\left((0, T), L^{\infty}(\mathbb{R})\right)\right]^{d}}\left\|\partial_{x} u^{\varepsilon}\right\|_{\left[L^{2}\left((0, T), L^{1}(\mathbb{R})\right)\right]^{d}} \\
& \leq 2 T^{\frac{1}{2}}\|\phi\|_{\left[L^{2}\left((0, T), W^{1, \infty}(\mathbb{R})\right)\right]^{d}}\left\|u_{0}\right\|_{\left[L^{\infty}(\mathbb{R})\right]^{d}},
\end{aligned}
$$

where we have used inequality (3.5). Similarly, for the term $I_{2}$, we have: 


$$
\begin{aligned}
\left|I_{2}\right| & \leq M_{0}\|\phi\|_{\left[L^{2}\left((0, T), L^{\infty}(\mathbb{R})\right)\right]^{d}}\left\|\partial_{x} u^{\varepsilon}\right\|_{\left[L^{2}\left((0, T), L^{1}(\mathbb{R})\right)\right]^{d}}, \\
& \leq 2 T^{\frac{1}{2}} M_{0}\left\|u_{0}\right\|_{\left[L^{\infty}(\mathbb{R})\right]^{d}}\|\phi\|_{\left[L^{2}\left((0, T), W^{1, \infty}(\mathbb{R})\right)\right]^{d}} .
\end{aligned}
$$

Finally, collecting (3.6) and (3.7), we get that there exists a constant $C=C\left(T, M_{0}\right)$ independent of $0<\varepsilon \leq 1$ such that:

$$
\left|\int_{(0, T) \times \mathbb{R}} \phi \cdot \partial_{t} u^{\varepsilon}\right| \leq C\left\|u_{0}\right\|_{\left[L^{\infty}(\mathbb{R})\right]^{d}}\|\phi\|_{\left[L^{2}\left((0, T), W^{1, \infty}(\mathbb{R})\right)\right]^{d}}
$$

which gives the stated result.

\section{Corollary 3.4. (E-uniform estimates)}

Assume (H1) and that the function $u_{0} \in\left[L^{\infty}(\mathbb{R})\right]^{d}$ satisfies (H3). Then for any $0<\varepsilon \leq 1$, the solution $u^{\varepsilon}$ of the system (2.2) given in Theorem 2.2 with initial data $u_{0}^{\varepsilon}=u_{0} * \eta_{\varepsilon}$, satisfies the following $\varepsilon$-uniform estimate for all $T>0$ :

$$
\left\|\partial_{x} u^{\varepsilon}\right\|_{\left[L^{\infty}\left((0,+\infty), L^{1}(\mathbb{R})\right)\right]^{d}}+\left\|u^{\varepsilon}\right\|_{\left[L^{\infty}((0,+\infty) \times \mathbb{R})\right]^{d}}+\left\|\partial_{t} u^{\varepsilon}\right\|_{\left[L^{2}\left((0, T) ; W^{-1,1}(\mathbb{R})\right)\right]^{d}} \leq C,
$$

where $C=C\left(T, M_{0},\left\|u_{0}\right\|_{\left[L^{\infty}(\mathbb{R})\right]^{d}}\right)$.

This Corollary is a straightforward consequence of Remark 2.1, Theorem 2.2, estimate (3.5) and Lemma 3.3.

\section{Passage to the limit and proof of Theorem 1.1}

In this section, we prove that the system (1.1)-(1.2) admits solutions $u$ in the distributional sense. They are the limits of $u^{\varepsilon}$ given by Theorem 2.2 when $\varepsilon \rightarrow 0$. To do this, we will justify the passage to the limit as $\varepsilon$ tends to 0 in the system $(2.2)$ by using some compactness tools that are presented first in a subsection.

\subsection{Preliminary results}

First, for all open interval $I$ of $\mathbb{R}$, we denote by

$$
L \log L(I)=\left\{f \in L^{1}(I) \text { such that } \int_{I}|f| \ln (e+|f|)<+\infty\right\} .
$$

Lemma 4.1. (Simon's Lemma)

Let $X, B, Y$ be three Banach spaces, such that we have the following injections

$X \hookrightarrow B$ with compact embedding and $B \hookrightarrow Y$ with continuous embedding.

Let $T>0$. If $\left(u^{\varepsilon}\right)_{\varepsilon}$ is a sequence such that

$$
\left\|u^{\varepsilon}\right\|_{L^{\infty}((0, T) ; X)}+\left\|\partial_{t} u^{\varepsilon}\right\|_{L^{q}((0, T) ; Y)} \leq C,
$$


where $q>1$ and $C$ is a constant independent of $\varepsilon$, then $\left(u^{\varepsilon}\right)_{\varepsilon}$ is relatively compact in $L^{p}((0, T) ; B)$ for all $1 \leq p<q$.

For the proof, see Simon [27, Corollary 4, Page 85].

In order to show the existence of a solution to system (1.1) in Subsection 4.2, we will apply this lemma to each scalar component of $u^{\varepsilon}$ in the particular case where $X=W^{1,1}(I), B=L^{1}(I)$ and $Y=W^{-1,1}(I):=\left(W_{0}^{1, \infty}(I)\right)^{\prime}$.

We denote by $K_{\exp }(I)$ the class of all measurable functions $u$ defined on $I$ for which

$$
\int_{I}\left(e^{|u|}-1\right)<+\infty
$$

The space $\operatorname{EXP}(I)=\left\{\mu u: \quad \mu \geq 0\right.$ and $\left.u \in K_{\exp }(I)\right\}$ is the linear hull of $K_{\text {exp }}(I)$. This space is supplemented with the following Luxemburg norm (see Adams [1, (13), Page 234] ):

$$
\|u\|_{E X P(I)}=\inf \left\{\lambda>0: \int_{I}\left(e^{\frac{|u|}{\lambda}}-1\right) \leq 1\right\} .
$$

Let us recall some useful properties of this space.

Lemma 4.2. (Generalized Hölder inequality, Adams [1, 8.11, Page 234]) Let $h \in \operatorname{EXP}(I)$ and $g \in L \log L(I)$. Then $h g \in L^{1}(I)$, with

$$
\|h g\|_{L^{1}(I)} \leq 2\|h\|_{E X P(I)}\|g\|_{L \log L(I)} .
$$

\section{Lemma 4.3. (Continuity)}

Let $T>0$. Assume that $u \in L^{\infty}((0,+\infty) \times \mathbb{R})$ such that

$$
\left\|\partial_{x} u\right\|_{L^{\infty}((0, T) ; L \log L(\mathbb{R}))}+\left\|\partial_{t} u\right\|_{L^{\infty}((0, T) ; L \log L(\mathbb{R}))} \leq C_{2}
$$

Then for all $\delta, h \geq 0$ and all $(t, x) \in(0, T-\delta) \times \mathbb{R}$, we have:

$$
|u(t+\delta, x+h)-u(t, x)| \leq 6 C_{2}\left(\frac{1}{\ln \left(\frac{1}{\delta}+1\right)}+\frac{1}{\ln \left(\frac{1}{h}+1\right)}\right) .
$$

\section{Proof of Lemma 4.3:}

For all $h>0$ and $(t, x) \in(0, T) \times \mathbb{R}$, we have: 


$$
\begin{aligned}
|u(t, x+h)-u(t, x)| & \leq\left|\int_{x}^{x+h} \partial_{x} u(t, y) d y\right| \\
& \leq 2\|1\|_{E X P(x, x+h)}\left\|\partial_{x} u(t, \cdot)\right\|_{L \log L(x, x+h)} \\
& \leq 2 \frac{1}{\ln \left(\frac{1}{h}+1\right)}\left\|\partial_{x} u\right\|_{L^{\infty}((0, T) ; L \log L(\mathbb{R}))}, \\
& \leq 2 C_{2} \frac{1}{\ln \left(\frac{1}{h}+1\right)}
\end{aligned}
$$

where we have used in the second line the generalized Hölder inequality (Lemma $4.2)$. Now we prove the continuity in time for all $\delta>0$ and $(t, x) \in(0, T-\delta) \times \mathbb{R}$, we have:

$$
\begin{aligned}
& \delta|u(t+\delta, x)-u(t, x)| \\
& =\int_{x}^{x+\delta}|u(t+\delta, x)-u(t, x)| d y, \\
& \leq \overbrace{\int_{x}^{x+\delta}|u(t+\delta, x)-u(t+\delta, y)| d y}^{K_{1}}+\overbrace{\int_{x}^{x+\delta}|u(t+\delta, y)-u(t, y)| d y}^{K_{2}}+\overbrace{\int_{x}^{x+\delta}|u(t, y)-u(t, x)| d y}^{K_{3}} .
\end{aligned}
$$

Similarly, as in the last estimate (4.1), we get that:

$$
\begin{aligned}
K_{1}+K_{3} & \leq \delta \int_{x}^{x+\delta}\left|\partial_{x} u(t+\delta, y)\right| d y,+\delta \int_{x}^{x+\delta}\left|\partial_{x} u(t, y)\right| d y, \\
& \leq 4 C_{2} \frac{\delta}{\ln \left(\frac{1}{\delta}+1\right)} .
\end{aligned}
$$

Now we use the fact that $\partial_{t} u$ is bounded in $L^{\infty}((0, T) ; L \log L(\mathbb{R}))$, to obtain that:

$$
\begin{aligned}
K_{2} & \leq \int_{x}^{x+\delta} \int_{t}^{t+\delta}\left|\partial_{t} u(s, y)\right| d s d y, \\
& \leq 2 \delta\|1\|_{E X P(x, x+\delta)}\left\|\partial_{t} u\right\|_{L^{\infty}((0, T) ; L \log L(\mathbb{R}))} \leq 2 C_{2} \frac{\delta}{\ln \left(\frac{1}{\delta}+1\right)} .
\end{aligned}
$$

Collecting the estimates of $K_{1}, K_{2}$ and $K_{3}$, we get that:

$$
|u(t+\delta, x)-u(t, x)| \leq \frac{1}{\delta}\left(K_{1}+K_{2}+K_{3}\right) \leq 6 C_{2} \frac{1}{\ln \left(\frac{1}{\delta}+1\right)} .
$$


This last inequality joint to (4.1) implies the result.

\subsection{Proof of Theorem 1.1}

The authors would like to thank T. Gallouët for fruitful remarks that helped to simplify the proof of Theorem 1.1. Before proving Theorem 1.1, we first prove the following result.

\section{Theorem 4.4. (Passage to the limit)}

Assume that $u^{\varepsilon}$ is a solution of system (2.2) given by Theorem 2.2, with initial data $u_{0}^{\varepsilon}=u_{0} * \eta_{\varepsilon}$ where $u_{0}$ satisfies (H3). If we assume that for all $T>0$, there exists a constant $C>0$ independent on $\varepsilon$, such that:

$$
\left\|\partial_{x} u^{\varepsilon}\right\|_{\left[L^{\infty}((0, T) ; L \log L(\mathbb{R}))\right]^{d}} \leq C,
$$

then up to extract a subsequence, the function $u^{\varepsilon}$ converges to a function $u$ weakly- $\star$ in $\left[L^{\infty}((0,+\infty) \times \mathbb{R})\right]^{d}$, as $\varepsilon$ goes to zero. Moreover, $u$ is a solution of (1.1)-(1.2), and satisfies

$$
\left\{\begin{array}{l}
\|u\|_{\left[L^{\infty}((0,+\infty) \times \mathbb{R})\right]^{d}} \leq\left\|u_{0}\right\|_{\left[L^{\infty}(\mathbb{R})\right]^{d}}, \\
\left\|\partial_{x} u\right\|_{\left[L^{\infty}((0, T) ; L \log L(\mathbb{R}))\right]^{d}} \leq C, \\
\left\|\partial_{t} u\right\|_{\left[L^{\infty}((0, T) ; L \log L(\mathbb{R}))\right]^{d}} \leq M_{0} C,
\end{array}\right.
$$

and $u(t, \cdot)$ is non-decreasing in $x$, for all $t>0$ and satisfies

$$
\left\|u^{i}\right\|_{L^{\infty}\left((0,+\infty) ; L^{1}(\mathbb{R})\right)} \leq 2\left\|u_{0}^{i}\right\|_{L^{\infty}(\mathbb{R})} \quad \text { for } \quad i=1, \ldots, d .
$$

\section{Proof of Theorem 4.4:}

Step 1 ( $u$ solution of (1.1)): First, we remark that with the estimate (3.8) we know that for any $T>0$, the solutions $u^{\varepsilon}$ of the system (2.2) obtained with the help of Theorem 2.2, are $\varepsilon$-uniformly bounded in $\left[L^{\infty}((0,+\infty) \times \mathbb{R})\right]^{d}$. Hence, as $\varepsilon$ goes to zero, we can extract a subsequence still denoted by $u^{\varepsilon}$, that converges weakly- $\star$ in $\left[L^{\infty}((0,+\infty) \times \mathbb{R})\right]^{d}$ to some limit $u$. Then we want to show that $u$ is a solution of system (1.1). Indeed, since the passage to the limit in the linear terms is trivial in $\left[\mathcal{D}^{\prime}((0,+\infty) \times \mathbb{R})\right]^{d}$, it suffices to pass to the limit in the non-linear term

$$
\lambda\left(u^{\varepsilon}\right) \diamond \partial_{x} u^{\varepsilon} .
$$

According to estimate (3.8) we know that for all open and bounded interval $I$ of $\mathbb{R}$ there exists a constant $C$ independent on $\varepsilon$ such that:

$$
\left\|u^{\varepsilon}\right\|_{\left[L^{\infty}\left((0, T) ; W^{1,1}(I)\right)\right]^{d}}+\left\|\partial_{t} u^{\varepsilon}\right\|_{\left[L^{2}\left((0, T) ; W^{-1,1}(I)\right)\right]^{d}} \leq C .
$$


From the compactness of $W^{1,1}(I) \hookrightarrow L^{1}(I)$, we can apply Simon's Lemma (i.e. Lemma 4.1), with $X=\left[W^{1,1}(I)\right]^{d}, B=\left[L^{1}(I)\right]^{d}$ and $Y=\left[W^{-1,1}(I)\right]^{d}$, which shows in particular that

$$
u^{\varepsilon} \text { is relatively compact in }\left[L^{1}((0, T) \times I)\right]^{d} .
$$

Then, we can see that (up to extract a subsequence)

$$
\lambda\left(u^{\varepsilon}\right) \rightarrow \lambda(u) \quad \text { a.e. }
$$

Moreover, from Lemma 4.2, similarly as in (4.1), we can get, for all $t \in(0, T)$ the following estimates:

$$
\left|\int_{I} \partial_{x} u^{\varepsilon}(t, y) d y\right| \leq 2 C \frac{1}{\ln \left(\frac{1}{|I|}+1\right)},
$$

where $C$ is given in (4.2). By the previous estimate and the fact that $\lambda\left(u^{\varepsilon}\right)$ is uniformly bounded in $\left[L^{\infty}((0,+\infty) \times \mathbb{R})\right]^{d}$ and converges a.e. to $\lambda(u)$, we can apply the Dunford-Pettis Theorem (see Brezis [4, Th IV.29]) and prove that

$$
\lambda\left(u^{\varepsilon}\right) \diamond \partial_{x} u^{\varepsilon} \rightarrow \lambda(u) \diamond \partial_{x} u
$$

weakly in $\left[L^{1}((0, T) \times I)\right]^{d}$. Because this is true for any bounded open interval $I$, then we can pass to the limit in (2.2) and get that,

$$
\partial_{t} u+\lambda(u) \diamond \partial_{x} u=0 \quad \text { in } \quad \mathcal{D}^{\prime}((0,+\infty) \times \mathbb{R}) .
$$

Step 2 (A priori bounds): By weakly- $\star$ convergence and from the fact that

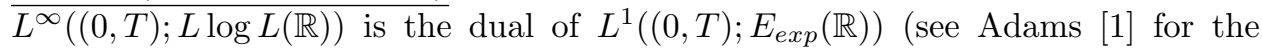
definition of the Banach space $\left.E_{\text {exp }}(\mathbb{R})\right)$, we can check that $u$ satisfies the following estimates:

$$
\begin{gathered}
\left\|\partial_{x} u\right\|_{\left[L^{\infty}((0, T) ; L \log L(\mathbb{R}))\right]^{d}} \leq \liminf _{\varepsilon \rightarrow 0}\left\|\partial_{x} u^{\varepsilon}\right\|_{\left[L^{\infty}((0, T) ; L \log L(\mathbb{R}))\right]^{d}} \leq C, \\
\|u\|_{\left[L^{\infty}((0,+\infty) \times \mathbb{R})\right]^{d}} \leq \liminf _{\varepsilon \rightarrow 0}\left\|u^{\varepsilon}\right\|_{\left[L^{\infty}((0,+\infty) \times \mathbb{R})\right]^{d}} \leq\left\|u_{0}\right\|_{\left[L^{\infty}(\mathbb{R})\right]^{d}} .
\end{gathered}
$$

Thanks to these two estimates, we obtain that

$$
\begin{aligned}
\left\|\partial_{t} u\right\|_{\left[L^{\infty}((0, T) ; L \log L(\mathbb{R}))\right]^{d}} & \leq\left\|\lambda(u) \diamond \partial_{x} u\right\|_{\left[L^{\infty}((0, T) ; L \log L(\mathbb{R}))\right]^{d}} \\
& \leq M_{0}\left\|\partial_{x} u\right\|_{\left[L^{\infty}((0, T) ; L \log L(\mathbb{R}))\right]^{d}} \leq M_{0} C .
\end{aligned}
$$

Moreover (4.3) follows from (4.5) and the fact that $u(t, \cdot)$ is non-decreasing in $x$ (as it was the case for $\left.u^{\varepsilon}\right)$. 
Step 3 (Recovering the initial data): Now we prove that the initial conditions (1.2) coincide with $u(0, \cdot)$. Indeed, by the $\varepsilon$-uniformly estimate given in Corollary 3.4 , we can prove easily that

$$
\left\|u^{\varepsilon}(t)-u_{0}^{\varepsilon}\right\|_{\left[W^{-1,1}(\mathbb{R})\right]^{d}} \leq C t^{\frac{1}{2}}
$$

Then, we get

$$
\begin{aligned}
\left\|u(t)-u_{0}\right\|_{\left[W^{-1,1}(\mathbb{R})\right]^{d}} & \leq\left\|u-u_{0}\right\|_{\left[L^{\infty}\left((0, t) ; W^{-1,1}(\mathbb{R})\right)\right]^{d}} \\
& \leq \liminf _{\varepsilon \rightarrow 0}\left\|u^{\varepsilon}-u_{0}^{\varepsilon}\right\|_{\left[L^{\infty}\left((0, t) ; W^{-1,1}(\mathbb{R})\right)\right]^{d}} \leq C t^{\frac{1}{2}},
\end{aligned}
$$

where we have used the weakly- $\star$ convergence in $L^{\infty}\left((0, t) ; W^{-1,1}(\mathbb{R})\right)$ in the second line. This proves that $u(0, \cdot)=u_{0}$ in $\left[\mathcal{D}^{\prime}(\mathbb{R})\right]^{d}$.

\section{Proof of Theorem 1.1:}

Step 1 (Existence): Note that with assumption $(\mathrm{H} 2)$ and estimate (3.2), we deduce from (3.4) joint to (3.5) that, the solution $u^{\varepsilon}$ given by Corollary 3.4 satisfies the following estimate:

$$
\left\|\partial_{x} u^{\varepsilon}\right\|_{\left[L^{\infty}((0, T) ; L \log L(\mathbb{R}))\right]^{d}} \leq C,
$$

where $C=C\left(T, d, M_{1},\left\|u_{0}\right\|_{\left[L^{\infty}(\mathbb{R})\right]^{d}},\left\|\partial_{x} u_{0}\right\|_{[L \log L(\mathbb{R})]^{d}}\right)$. Now, we apply Theorem 4.4 to prove that, up to extract a subsequence, the function $u^{\varepsilon}$ converges, as $\varepsilon$ goes to zero, to a function $u$ weakly- $\star$ in $\left[L^{\infty}((0,+\infty) \times \mathbb{R})\right]^{d}$, with $u$ being the solution to (1.1)-(1.2). Moreover, from Lemma 4.3, we deduce that the function $u$ satisfies the continuity estimate (1.7).

\section{Step 2 (Justification of (1.5)): Let}

$$
\left\{\begin{array}{l}
\Gamma_{i j}\left(u^{\varepsilon}\right)=\frac{1}{2}\left(\lambda_{, j}^{i}\left(u^{\varepsilon}\right)+\lambda_{, i}^{j}\left(u^{\varepsilon}\right)\right), \quad \text { for } \quad i, j=1, \ldots, d, \\
w^{\varepsilon}=\partial_{x} u^{\varepsilon} .
\end{array}\right.
$$

For a general matrix $\Gamma$, where ${ }^{t} \Gamma=\Gamma \geq 0$, let us introduce the square root $B=\sqrt{\Gamma}$ of $\Gamma$, uniquely defined by

$$
{ }^{t} B=B \geq 0 \quad \text { and } \quad B^{2}=\Gamma .
$$

Note that for non-negative symmetric matrices, the map $\Gamma \longmapsto \sqrt{\Gamma}$ is continuous. Then we can write 


$$
\int_{0}^{t} \int_{\mathbb{R}_{i, j=1, \ldots, d}} \lambda_{, j}^{i}\left(u^{\varepsilon}\right) w^{\varepsilon, i} w^{\varepsilon, j}=\int_{0}^{t} \int_{\mathbb{R}}\left|\sqrt{\Gamma\left(u^{\varepsilon}\right)} w^{\varepsilon}\right|^{2} \leq C,
$$

where $C$ is given in (3.2). Therefore

$$
\sqrt{\Gamma\left(u^{\varepsilon}\right)} w^{\varepsilon} \rightarrow q \text { weakly in }\left[L^{2}((0, t) \times \mathbb{R})\right]^{d} .
$$

Applying the same argument as in Step 1, of the proof of Theorem 4.4, for the convergence of $\lambda\left(u^{\varepsilon}\right) \diamond \partial_{x} u^{\varepsilon}$, we see that

$$
\sqrt{\Gamma\left(u^{\varepsilon}\right)} \partial_{x} u^{\varepsilon} \rightarrow \sqrt{\Gamma(u)} \partial_{x} u=q \text { weakly in }\left[L^{1}((0, t) \times \mathbb{R})\right]^{d} .
$$

Therefore, using the weakly convergence in $L^{2}((0, t) \times \mathbb{R})$, we get

$$
\int_{0}^{t} \int_{\mathbb{R}_{i, j=1, \ldots, d}} \lambda_{, j}^{i}(u) \partial_{x} u^{i} \partial_{x} u^{j}=\int_{0}^{t} \int_{\mathbb{R}} q^{2} \leq \liminf _{\varepsilon \rightarrow 0} \int_{0}^{t} \int_{\mathbb{R}}\left|\sqrt{\Gamma\left(u^{\varepsilon}\right)} \partial_{x} u^{\varepsilon}\right|^{2} \leq C .
$$

Remark also that for $w^{i}=\partial_{x} u^{i}$, we have

$$
\begin{aligned}
\sup _{0 \leq t \leq T} \int_{\mathbb{R}} f\left(w^{i}\right) & \leq 1+\left\|w^{i}\right\|_{L^{\infty}((0, T) ; L \log L(\mathbb{R}))}+\left\|w^{i}\right\|_{L^{\infty}\left((0, T) ; L^{1}(\mathbb{R})\right)} \ln \left(1+\left\|w^{i}\right\|_{L^{\infty}((0, T) ; L \log L(\mathbb{R}))}\right) \\
& \leq 1+\left\|w^{i}\right\|_{L^{\infty}((0, T) ; L \log L(\mathbb{R}))}+2\left\|u_{0}^{i}\right\|_{L^{\infty}(\mathbb{R})} \ln \left(1+\left\|w^{i}\right\|_{L^{\infty}((0, T) ; L \log L(\mathbb{R}))}\right):=g\left[w^{i}\right] \\
& \leq \liminf _{\varepsilon \rightarrow 0} g\left[w^{\varepsilon, i}\right] \\
& \leq 1+C+2\left\|u_{0}^{i}\right\|_{L^{\infty}(\mathbb{R})} \ln (1+C):=C^{\prime},
\end{aligned}
$$

where in the first line we have used (3.3), in the second line we have used (4.3), in the third line we have used the weakly- $\star$ convergence of $w^{\varepsilon, i}$ towards $w^{i}$ in $L^{\infty}((0, T) ; L \log L(\mathbb{R}))$ and in the fourth line, we have used (4.6). Putting this result together with (4.7), we get (1.5) with $C_{1}=C+C^{\prime}$.

\section{Appendix: Example of the dynamics of dislocation densities}

In this section, we present a model describing the dynamics of dislocation densities. We refer to Hirth et al. [16] for a physical presentation of dislocations which are (moving) defects in crystals. Even if the problem is naturally a three-dimensional problem, we will first assume that the geometry of the problem is invariant by translations in the $x_{3}$-direction. This reduces the problem to the study of dislocations densities defined on the plane $\left(x_{1}, x_{2}\right)$ and moving in a given direction $b$ belonging 
to the plane $\left(x_{1}, x_{2}\right)$ (which is called "Burger's vector").

In Subsection 5.1, we present the 2D-model with multi-slip directions. In the particular geometry where the dislocations densities only depend on the variable $x=x_{1}+x_{2}$, this two-dimensional model reduces to a one-dimensional model which is presented in Subsection 5.2. Finally in Subsection 5.3, we explain how to recover equation (1.1) as a model for dislocation dynamics with

$$
\lambda^{i}(u)=\sum_{j=1, \ldots, d} A_{i j} u^{j}
$$

for some particular non-negative and symmetric matrix $A$.

\subsection{The 2D-model}

We now present in detail the two-dimensional model. We denote by $X$ the vector $X=\left(x_{1}, x_{2}\right) \in \mathbb{R}^{2}$. We consider a crystal filling the whole space $\mathbb{R}^{2}$ and its displacement $v=\left(v_{1}, v_{2}\right): \mathbb{R}^{2} \rightarrow \mathbb{R}^{2}$, where we have not yet introduced any time dependence.

We introduce the total strain $\varepsilon(v)=\left(\varepsilon_{i j}(v)\right)_{i, j=1,2}$ which is a symmetric matrix defined by

$$
\varepsilon_{i j}(v)=\frac{1}{2}\left(\frac{\partial v_{i}}{\partial x_{j}}+\frac{\partial v_{j}}{\partial x_{i}}\right) .
$$

The total strain can be split into two parts:

$$
\varepsilon_{i j}(v)=\varepsilon_{i j}^{e}+\varepsilon_{i j}^{p} \quad \text { with } \quad \varepsilon^{p}=\sum_{k=1, \ldots, d} \varepsilon^{0, k} u^{k},
$$

where $\varepsilon_{i j}^{e}$ is the elastic strain and $\varepsilon_{i j}^{p}$ is the plastic strain. The scalar function $u^{k}$ is the plastic displacement associated to the $k$-th slip system whose matrix $\varepsilon_{i j}^{0, k}$ is defined by

$$
\varepsilon_{i j}^{0, k}=\frac{1}{2}\left(b_{i}^{k} n_{j}^{k}+n_{i}^{k} b_{j}^{k}\right),
$$

where $\left(b^{k}, n^{k}\right)$ is a family of vectors in $\mathbb{R}^{2}$, such that $n^{k}$ is a unit vector orthogonal to the Burger's vector $b^{k}$ (see Hirth et al. [16] for the definition of Burger's vector of a dislocation)

To simplify the presentation, we assume the simplest possible periodicity property of the unknowns. 
Assumption (H):

i) The function $v$ is $\mathbb{Z}^{2}$-periodic with $\int_{(0,1)^{2}} v d X=0$.

ii) For each $k=1, \ldots, d$, there exists $L^{k} \in \mathbb{R}^{2}$ such that $u^{k}(X)-L^{k} \cdot X$ is a $\mathbb{Z}^{2}$ periodic.

iii) The integer $d$ is even with $d=2 N$ and we have for $k=1, \ldots, N$ :

$$
L^{k+N}=L^{k}, n^{k+N}=n^{k}, \quad b^{k+N}=-b^{k}, \quad \varepsilon^{0, k+N}=-\varepsilon^{0, k} .
$$

iv) We denote by $\tau^{k} \in \mathbb{R}^{2}$ a unit vector parallel to $b^{k}$ such that $\tau^{k+N}=\tau^{k}$. We require that $L^{k}$ is chosen such $\tau^{k} \cdot L^{k} \geq 0$.

Note in particular that the plastic strain $\varepsilon_{i j}^{p}$ is $\mathbb{Z}^{2}$-periodic as a consequence of Assumption $(H)$. The stress matrix is then given by

$$
\sigma_{i j}=\sum_{k, l=1,2} \Lambda_{i j k l} \varepsilon_{k l}^{e} \quad \text { for } \quad i, j=1,2,
$$

where $\Lambda=\left(\Lambda_{i j k l}\right)_{i, j, k, l=1,2}$, are the constant elastic coefficients of the material, satisfying for some constant $m>0$ :

$$
\sum_{i, j, k, l=1,2} \Lambda_{i j k l} \varepsilon_{i j} \varepsilon_{k l} \geq m \sum_{i, j=1,2} \varepsilon_{i j}^{2},
$$

for all symmetric matrices $\varepsilon=\left(\varepsilon_{i j}\right)_{i j}$, i.e. such that $\varepsilon_{i j}=\varepsilon_{j i}$.

Then the stress is assumed to satisfy the equation of elasticity

$$
\sum_{j=1,2} \frac{\partial \sigma_{i j}}{\partial x_{j}}=0 \quad \text { for } \quad i=1,2 .
$$

On the other hand the plastic displacement $u^{k}$ is assumed to satisfy the following transport equation

$$
\partial_{t} u^{k}=c^{k} \tau^{k} . \nabla u^{k} \quad \text { with } \quad c^{k}=\sum_{i, j=1,2} \sigma_{i j} \varepsilon_{i j}^{0, k} .
$$

This equation can be interpreted, by observing that

$$
\theta^{k}=\tau^{k} \cdot \nabla u^{k} \geq 0,
$$

is the density of edge dislocations associated to the Burger's vector $b^{k}$ moving in the direction $\tau^{k}$ at the velocity $c^{k}$. Here $c^{k}$ is also called the resolved Peach-Koehler 
force in the physical literature. In particular, we see that the dislocation density $\theta^{k}$ satisfies the following conservation law

$$
\partial_{t} \theta^{k}=\operatorname{div}\left(c^{k} \tau^{k} \theta^{k}\right) .
$$

Finally, for $k=1, \ldots, d$, the functions $u^{k}$ and $v$ are then assumed to depend on $(t, X) \in(0,+\infty) \times \mathbb{R}^{2}$ and to be solutions of the coupled system (see Yefimov [29, ch. 5.] and Yefimov, Van der Giessen [30]):

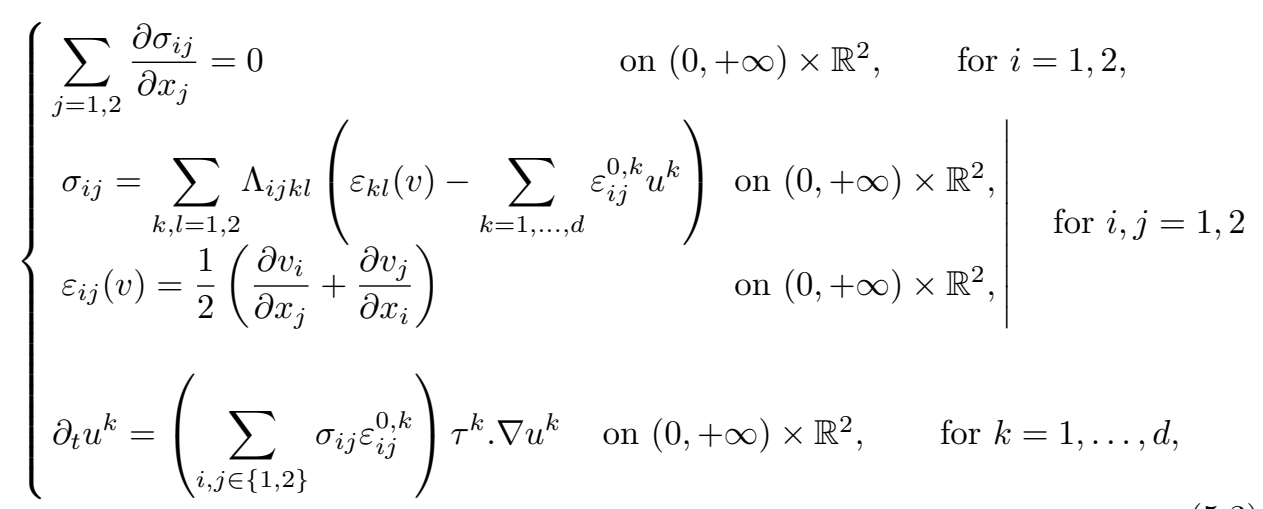

where $\Lambda_{i j k l}, \varepsilon_{i j}^{0, k}$ are fixed parameters previously introduced, and the unknowns of the system are $u=\left(u^{k}\right)_{k=1, \ldots, d}$ and the displacement $v=\left(v_{1}, v_{2}\right)$. Note also that these equations are compatible with the periodicity assumptions $(H),(i)-(i i)$.

For a detailed physical presentation of a model with multi-slip directions, we refer to Yefimov, Van der Giessen [30] and Yefimov [29, ch. 5.] and to Groma, Balogh [15] for the case of a model with a single slip direction. See also Cannone et al. [5] for a mathematical analysis of the Groma, Balogh model.

\subsection{Derivation of the 1D-model}

In this subsection we are interested in a particular geometry where the dislocation densities depend only on the variable $x=x_{1}+x_{2}$. This will lead to a 1D-model. More precisely, we assume the following:

Assumption $\left(H^{\prime}\right)$ :

i) The functions $v(t, X)$ and $u^{k}(t, X)-L^{k} \cdot X$ depend only on the variable $x=x_{1}+x_{2}$.

ii) For $k=1, \ldots, d$, the vector $\tau^{k}=\left(\tau_{1}^{k}, \tau_{2}^{k}\right)$ satisfies $\tau_{1}^{k}+\tau_{2}^{k}>0$ with $\mu^{k}=\frac{1}{\tau_{1}^{k}+\tau_{2}^{k}}$.

iii) For $k=1, \ldots, d$, the vector $L^{k}=\left(L_{1}^{k}, L_{2}^{k}\right)$ satisfies $L_{1}^{k}=L_{2}^{k}=l^{k}$. 
For this particular one-dimensional geometry, we denote by an abuse of notation the function $v=v(t, x)$ which is 1-periodic in $x$. By assumption $\left(H^{\prime}\right),(i i i)$, we can see (again by an abuse of notation) that $u=\left(u^{k}(t, x)\right)_{k=1, \ldots, d}$ is such that for $k=1, \ldots, d, u^{k}(t, x)-l^{k} \cdot x$ is 1 -periodic in $x$.

Now, we can integrate the equations of elasticity, i.e. the first equation of (5.3). Using the $\mathbb{Z}^{2}$-periodicity of the unknowns (see assumption $(H),(i)$ - $(i i)$ ), and the fact that $\varepsilon^{0, k+N}=-\varepsilon^{0, k}$ (see assumption $(H),(i i i)$ ), we can easily conclude that the strain

$\varepsilon^{e}$ is a linear function of $\left(u^{j}-u^{j+N}\right)_{j=1, \ldots, N}$ and of $\left(\int_{0}^{1}\left(u^{j}-u^{j+N}\right) d x\right)_{j=1, \ldots, N}$.

This leads to the following Lemma

Lemma 5.1. (Stress for the 1D-model)

Under assumptions $(H),(i)-(i i)-(i i i)$ and $\left(H^{\prime}\right),(i)-(i i i)$ and (5.1), we have

$$
-\sigma: \varepsilon^{0, i}=\sum_{j=1, \ldots, d} A_{i j} u^{j}+\sum_{j=1, \ldots, d} Q_{i j} \int_{0}^{1} u^{j} d x, \quad \text { for } i=1, \ldots, N,
$$

where for $i, j=1, \ldots, N$

$$
\left\{\begin{array}{l}
A_{i, j}=A_{j, i} \quad \text { and } \quad A_{i+N, j}=-A_{i, j}=A_{i, j+N}=-A_{i+N, j+N} \\
Q_{i, j}=Q_{j, i} \quad \text { and } \quad Q_{i+N, j}=-Q_{i, j}=Q_{i, j+N}=-Q_{i+N, j+N} .
\end{array}\right.
$$

Moreover the matrix $A$ is non-negative.

The proof of Lemma 5.1 will be given at the end of this subsection.

Finally using Lemma 5.1, we can eliminate the stress and reduce the problem to a one-dimensional system of $d$ transport equations only depending on the function $u^{i}$, for $i=1, \ldots, d$. Naturally, from $(5.5)$ and $\left(H^{\prime}\right),(i i)$ this $1 \mathrm{D}$-model has the following form

\section{The 1D-model of the dynamics of dislocation densities:}

$\mu^{i} \partial_{t} u^{i}+\left(\sum_{j=1, \ldots, d} A_{i j} u^{j}+\sum_{j=1, \ldots, d} Q_{i j} \int_{0}^{1} u^{j} d x\right) \partial_{x} u^{i}=0, \quad$ on $(0,+\infty) \times \mathbb{R}, \quad$ for $i=1, \ldots, d$,

with from (5.2)

$$
\partial_{x} u^{i} \geq 0 \quad \text { for } i=1, \ldots, d
$$


Now, we give the proof of Lemma 5.1.

\section{Proof of Lemma 5.1:}

For the 2D-model, let us consider the elastic energy on the periodic cell (using the fact that $\varepsilon^{e}$ is $\mathbb{Z}^{2}$-periodic)

$$
E(u, v)=\frac{1}{2} \int_{(0,1)^{2}} \sum_{i, j, k, l=1,2} \Lambda_{i j k l} \varepsilon_{i j}^{e} \varepsilon_{k l}^{e} d X \quad \text { with } \quad \varepsilon_{i j}^{e}=\varepsilon_{i j}(v)-\sum_{k=1, \ldots, d} \varepsilon_{i j}^{0, k} u^{k} .
$$

By definition of $\sigma_{i j}$ and $\varepsilon_{i j}^{e}$, we have for $k=1, \ldots, d$

$$
\sum_{i, j=1,2}\left(\sigma_{i j} \varepsilon_{i j}^{0, k}\right)=-E_{u^{k}}^{\prime}(u, v) .
$$

On the other hand using $\left(H^{\prime}\right),(i)$-(iii), (with $x=x_{1}+x_{2}$ ) we can verify that we can rewrite the elastic energy as

$$
E=\frac{1}{2} \int_{0}^{1} \sum_{i, j, k, l=1,2} \Lambda_{i j k l} \varepsilon_{i j}^{e} \varepsilon_{k l}^{e} d x
$$

Replacing $\varepsilon_{i j}^{e}$ by its expression (5.4), we find that:

$$
\begin{aligned}
E= & \frac{1}{2} \int_{0}^{1} \sum_{i, j=1, \ldots, N} A_{i j}\left(u^{j}-u^{j+N}\right)\left(u^{i}-u^{i+N}\right) d x \\
& +\frac{1}{2} \sum_{i, j=1, \ldots, N} Q_{i j}\left(\int_{0}^{1}\left(u^{j}-u^{j+N}\right) d x\right)\left(\int_{0}^{1}\left(u^{i}-u^{i+N}\right) d x\right),
\end{aligned}
$$

for some symmetric matrices $A_{i j}=A_{j i}, Q_{i j}=Q_{j i}$. In particular, joint to (5.9) this gives exactly (5.5) with (5.6).

Let us now consider the functions $w^{i}=u^{i}-u^{i+N}$ such that

$$
\int_{0}^{1} w^{i} d x=0 \quad \text { for } \mathrm{i}=1, \ldots, \mathrm{N} .
$$

From (5.1), we deduce that

$$
0 \leq E=\frac{1}{2} \int_{0}^{1} \sum_{i, j=1, \ldots, N} A_{i j} w^{i} w^{j} d x
$$

More precisely, for all $i=1, \ldots, N$ and for all $\bar{w}^{i} \in \mathbb{R}$, we set

$$
w^{i}=\left\{\begin{array}{lll}
\bar{w}^{i} & \text { on } & {\left[0, \frac{1}{2}\right],} \\
-\bar{w}^{i} & \text { on } & {\left[\frac{1}{2}, 1\right]}
\end{array}\right.
$$


which satisfies (5.10). Finally, we obtain that

$$
0 \leq E=\frac{1}{2} \int_{0}^{1} \sum_{i, j=1, \ldots, N} A_{i j} \bar{w}^{i} \bar{w}^{j} d x .
$$

Because this is true for every $\bar{w}=\left(\bar{w}^{1}, \ldots, \bar{w}^{N}\right) \in \mathbb{R}^{N}$, we deduce that $A$ a nonnegative matrix.

We refer the reader to El Hajj [9] and El Hajj, Forcadel [10] for a study in the special case of a single slip direction, i.e. in the case $N=1$.

\subsection{Heuristic derivation of the non-periodic model}

Starting from the model (5.7)-(5.8) where for $i=1, \ldots, d$, the function $u^{i}(t, x)-l^{i} \cdot x$ is 1-periodic in $x$, we now want to rescale the unknowns to make the periodicity disappear. More precisely, we have the following Lemma:

\section{Lemma 5.2. (Non-periodic model)}

Let $u$ be a solution of (5.7)-(5.8) assuming Lemma 5.1 and $u^{i}(t, x)-l^{i} \cdot x$ is 1-periodic in $x$. Let

$$
u_{\delta}^{j}(t, x)=u^{j}(\delta t, \delta x), \quad \text { for a small } \delta>0 \text { and for } j=1, \ldots, d,
$$

such that, for all $j=1, \ldots, d$

$$
u_{\delta}^{j}(0, \cdot) \rightarrow \bar{u}^{j}(0, \cdot), \quad \text { as } \quad \delta \rightarrow 0, \quad \text { and } \quad \bar{u}^{j}(0, \pm \infty)=\bar{u}^{j+N}(0, \pm \infty) .
$$

Then $\bar{u}=\left(\bar{u}^{j}\right)_{j=1, \ldots, d}$ is formally a solution of

$$
\mu^{i} \partial_{t} \bar{u}^{i}+\left(\sum_{j=1, \ldots, d} A_{i j} \bar{u}^{j}\right) \partial_{x} \bar{u}^{i}=0, \quad \text { on }(0,+\infty) \times \mathbb{R},
$$

where the symmetric matrix $A$ is non-negative and $\partial_{x} \bar{u}^{i} \geq 0$ for $i=1, \ldots, d$.

We remark that the limit problem (5.12) is of type (1.1) when $\mu^{i}=1$. In particular, there are no reasons to assume that this system is strictly hyperbolic in general. Nevertheless, the general case $\mu^{i}>0$ can be treated with our approach developed in Theorem 1.1 considering the entropy $\int_{\mathbb{R}} \sum_{i=1, \ldots, d} \mu^{i} f\left(\partial_{x} \bar{u}^{i}(t, x)\right) d x$ instead of $\int_{\mathbb{R}} \sum_{i=1, \ldots, d} f\left(\partial_{x} \bar{u}^{i}(t, x)\right) d x$.

\section{Formal proof of Lemma 5.2:}

Here, we know that $u_{\delta}^{i}-\delta l^{i} \cdot x$ is $\frac{1}{\delta}$-periodic in $x$, and satisfies for $i=1, \ldots, d$ 


$$
\mu^{i} \partial_{t} u_{\delta}^{i}+\left(\sum_{j=1, \ldots, d} A_{i j} u_{\delta}^{j}+\delta \sum_{j=1, \ldots, d} Q_{i j} \int_{0}^{\frac{1}{\delta}} u_{\delta}^{j} d x\right) \partial_{x} u_{\delta}^{i}=0, \quad \text { on }(0,+\infty) \times \mathbb{R} .
$$

To simplify, assume that the initial data $u_{\delta}(0, \cdot)$ converge to a function $\bar{u}(0, \cdot)$ such that the function $\partial_{x} u_{\delta}(0, \cdot)$ inside the interval $\left(\frac{-1}{2 \delta}, \frac{1}{2 \delta}\right)$ has a support in $(-R, R)$, uniformly in $\delta$, where $R$ a positive constant. Because of the antisymmetry property of the matrix $Q$ (see assumption (5.6)), and because of assumption (5.11), we expect heuristically that the velocity in (5.13) remains uniformly bounded as $\delta \rightarrow 0$.

Therefore, using the finite propagation speed, we see that, there exists a constant $C$ independent in $\delta$, such that $\partial_{x} u_{\delta}(t, \cdot)$ has a support on $(-R-C t, R+C t) \subset\left(\frac{-1}{2 \delta}, \frac{1}{2 \delta}\right)$. Moreover, from (5.11) and the fact that

$$
\sum_{j=1, \ldots, d} Q_{i j} \int_{0}^{\frac{1}{\delta}} u_{\delta}^{j} d x=\sum_{j=1, \ldots, N} Q_{i j} \int_{0}^{\frac{1}{\delta}}\left(u_{\delta}^{j}-u_{\delta}^{j+N}\right) d x,
$$

we deduce that

$$
\sum_{j=1, \ldots, d} Q_{i j} \int_{0}^{\frac{1}{\delta}} u_{\delta}^{j} d x
$$

remains bounded uniformly in $\delta$. Then formally the non-local term vanishes and we get for $i=1, \ldots, d$

$$
\sum_{j=1, \ldots, d} A_{i j} u_{\delta}^{j}+\delta \sum_{j=1, \ldots, d} Q_{i j} \int_{0}^{\frac{1}{\delta}} u_{\delta}^{j} d x \rightarrow \sum_{j=1, \ldots, d} A_{i j} \bar{u}^{j}, \quad \text { as } \quad \delta \rightarrow 0,
$$

which proves that $\bar{u}$ is solution of (5.12), with the non-negative symmetric matrix A.

\section{Acknowledgements}

The first author would like to thank M. Cannone, T. Gallouët and M. Jazar for fruitful remarks that helped in the preparation of the paper. This work was partially supported the program "PPF, programme pluri-formations mathématiques financières et EDP", (2006-2010), Marne-la-Vallée University and École Nationale des Ponts et Chaussées and the ANR MICA "Mouvements d'Interfaces, Calcul et Applications" (2006-2009). 


\section{References}

[1] R. A. Adams, Sobolev spaces, Academic Press [A subsidiary of Harcourt Brace Jovanovich, Publishers], New York-London, 1975. Pure and Applied Mathematics, Vol. 65 .

[2] L. Ambrosio, Transport equation and Cauchy problem for BV vector fields, Invent. Math., 158 (2004), pp. 227-260.

[3] S. Bianchini AND A. BREssan, Vanishing viscosity solutions of nonlinear hyperbolic systems, Ann. of Math. (2), 161 (2005), pp. 223-342.

[4] H. Brezis, Analyse fonctionnelle, Collection Mathématiques Appliquées pour la Maîtrise. [Collection of Applied Mathematics for the Master's Degree], Masson, Paris, 1983. Théorie et applications. [Theory and applications].

[5] M. Cannone, A. El Haju, R. Monneau, and F. Ribaud, Global existence for a system of non-linear and non-local transport equations describing the dynamics of dislocation densities, to appear in Archive for Rational Mechanics and Analysis, (2007).

[6] R. J. DiPerna, Convergence of approximate solutions to conservation laws, Arch. Rational Mech. Anal., 82 (1983), pp. 27-70.

[7] R. J. DiPerna, Compensated compactness and general systems of conservation laws, Trans. Amer. Math. Soc., 292 (1985), pp. 383-420.

[8] R. J. DiPerna And P.-L. Lions, Ordinary differential equations, transport theory and Sobolev spaces, Invent. Math., 98 (1989), pp. 511-547.

[9] A. EL HAJJ, Well-posedness theory for a nonconservative Burgers-type system arising in dislocation dynamics, SIAM J. Math. Anal., 39 (2007), pp. 965-986.

[10] A. El HajJ And N. ForCAdel, A convergent scheme for a non-local coupled system modelling dislocations densities dynamics, Math. Comp., 77 (2008), pp. 789-812.

[11] A. El HajJ And R. Monneau, Some uniqueness results for diagonal hyperbolic systems with large and monotone data, In preparation, (2009).

[12] R. Eymard, T. Galloü̈T, And R. Herbin, Existence and uniqueness of the entropy solution to a nonlinear hyperbolic equation, Chinese Ann. Math. Ser. B, 16 (1995), pp. 1-14. A Chinese summary appears in Chinese Ann. Math. Ser. A 16 (1995), no. $1,119$.

[13] L. GȦRDing, Problème de Cauchy pour les systèmes quasi-linéaires d'ordre un strictement hyperboliques, in Les Équations aux Dérivées Partielles (Paris, 1962), Éditions du Centre National de la Recherche Scientifique, Paris, 1963, pp. 33-40.

[14] J. Glimm, Solutions in the large for nonlinear hyperbolic systems of equations, Comm. Pure Appl. Math., 18 (1965), pp. 697-715.

[15] I. Groma And P. BAlogh, Investigation of dislocation pattern formation in a twodimensional self-consistent field approximation, Acta Mater, 47 (1999), pp. 36473654 .

[16] J. P. Hirth And J. Lothe, Theory of dislocations, Second edition, Krieger, Malabar, Florida, 1992.

[17] S. N. KRUŽKov, First order quasilinear equations with several independent variables., Mat. Sb. (N.S.), 81 (123) (1970), pp. 228-255.

[18] P. D. LAX, Hyperbolic systems of conservation laws and the mathematical theory of shock waves, Society for Industrial and Applied Mathematics, Philadelphia, Pa., 1973. Conference Board of the Mathematical Sciences Regional Conference Series in Applied Mathematics, No. 11.

[19] P. LeFloch, Entropy weak solutions to nonlinear hyperbolic systems under nonconservative form, Comm. Partial Differential Equations, 13 (1988), pp. 669-727.

[20] P. LEFLOCH AND T.-P. LiU, Existence theory for nonlinear hyperbolic systems in 
nonconservative form, Forum Math., 5 (1993), pp. 261-280.

[21] P. G. LeFLOCH, Graph solutions of nonlinear hyperbolic systems, J. Hyperbolic Differ. Equ., 1 (2004), pp. 643-689.

[22] G. M. Lieberman, Second order parabolic differential equations, World Scientific Publishing Co. Inc., River Edge, NJ, 1996.

[23] P.-L. Lions, B. Perthame, And E. TAdmor, A kinetic formulation of multidimensional scalar conservation laws and related equations, J. Amer. Math. Soc., 7 (1994), pp. 169-191.

[24] O. A. OleINIK, Discontinuous solutions of non-linear differential equations, Amer. Math. Soc. Transl. (2), 26 (1963), pp. 95-172.

[25] F. Poupaud, Global smooth solutions of some quasi-linear hyperbolic systems with large data, Ann. Fac. Sci. Toulouse Math. (6), 8 (1999), pp. 649-659.

[26] D. Serre, Systems of conservation laws. I, II, Cambridge University Press, Cambridge, 1999-2000. Geometric structures, oscillations, and initial-boundary value problems, Translated from the 1996 French original by I. N. Sneddon.

[27] J. Simon, Compact sets in the space $L^{p}(0, T ; B)$, Ann. Mat. Pura Appl. (4), 146 (1987), pp. 65-96.

[28] L. TARTAR, Compensated compactness and applications to partial differential equations, in Nonlinear analysis and mechanics: Heriot-Watt Symposium, Vol. IV, vol. 39 of Res. Notes in Math., Pitman, Boston, Mass., 1979, pp. 136-212.

[29] S. Yefimov, Discrete dislocation and nonlocal crystal plasticity modelling, Netheerlands Institute for Metals Research, University of Groningen, 2004.

[30] S. Yefimov And E. VAN DeR Giessen, Multiple slip in a strain-gradient plasticity model motivated by a statistical-mechanics description of dislocations, International Journal of Solids and Structure, 42 (2005), pp. 3375-3394. 